\title{
$\operatorname{con} 5-841246 \cdot-10$
}

\section{MATERIALS ENGINEERING FOR TFCX}

\author{
DECLATMER
}

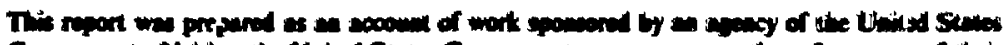

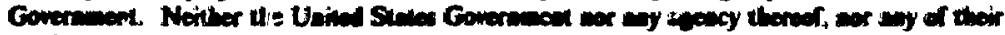

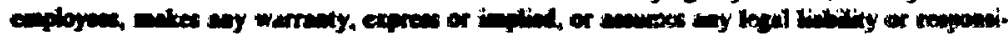

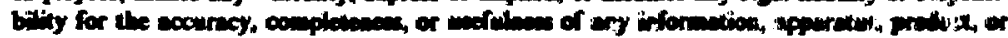

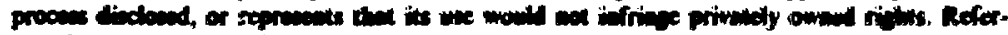

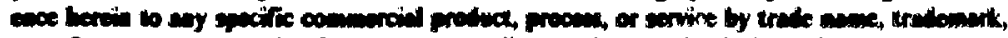

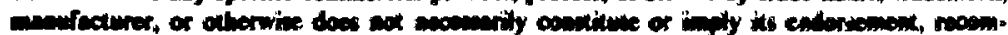

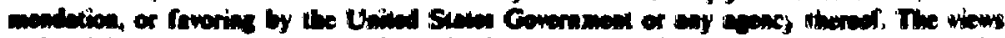

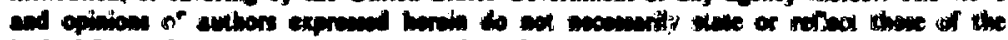

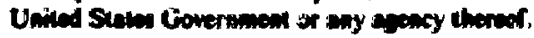

\section{F. W. Wiffen FEDC/ORNL}

\section{Oak Ridge, Tennesses}

\section{ICFRM-1 \\ Tokyo, Japan

* Research sponsored by the Office of Fusion Energy, U.S. Departwent of Enerty, under contract DE-ACO5-840R21400 with Martin Marietta Energy Systems, Inco-porated. 


\section{TFCX}

NOMINAL SUPERCONDUCTING OPTION

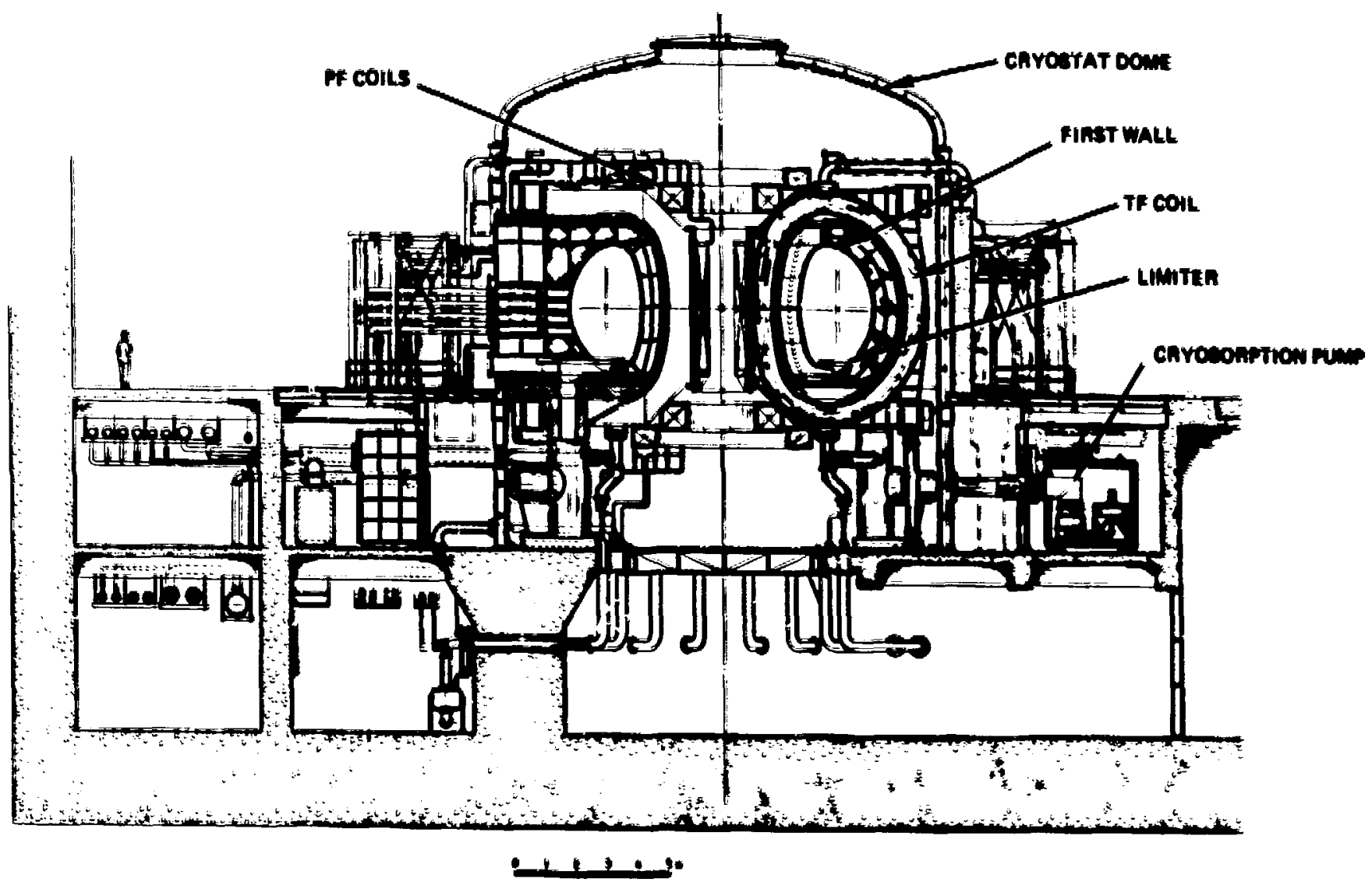




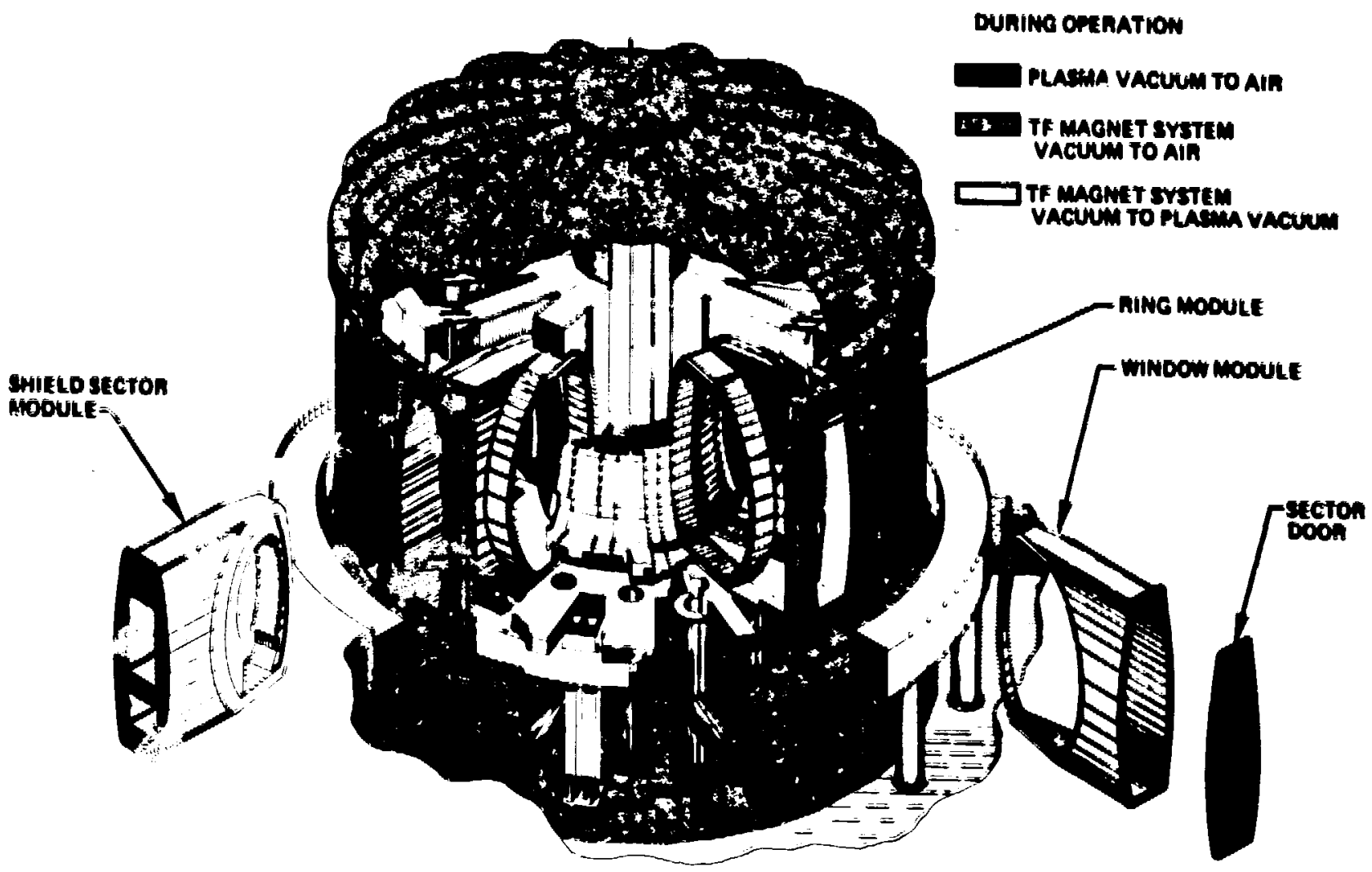

TFCX-S VACUUM INTERFACES 

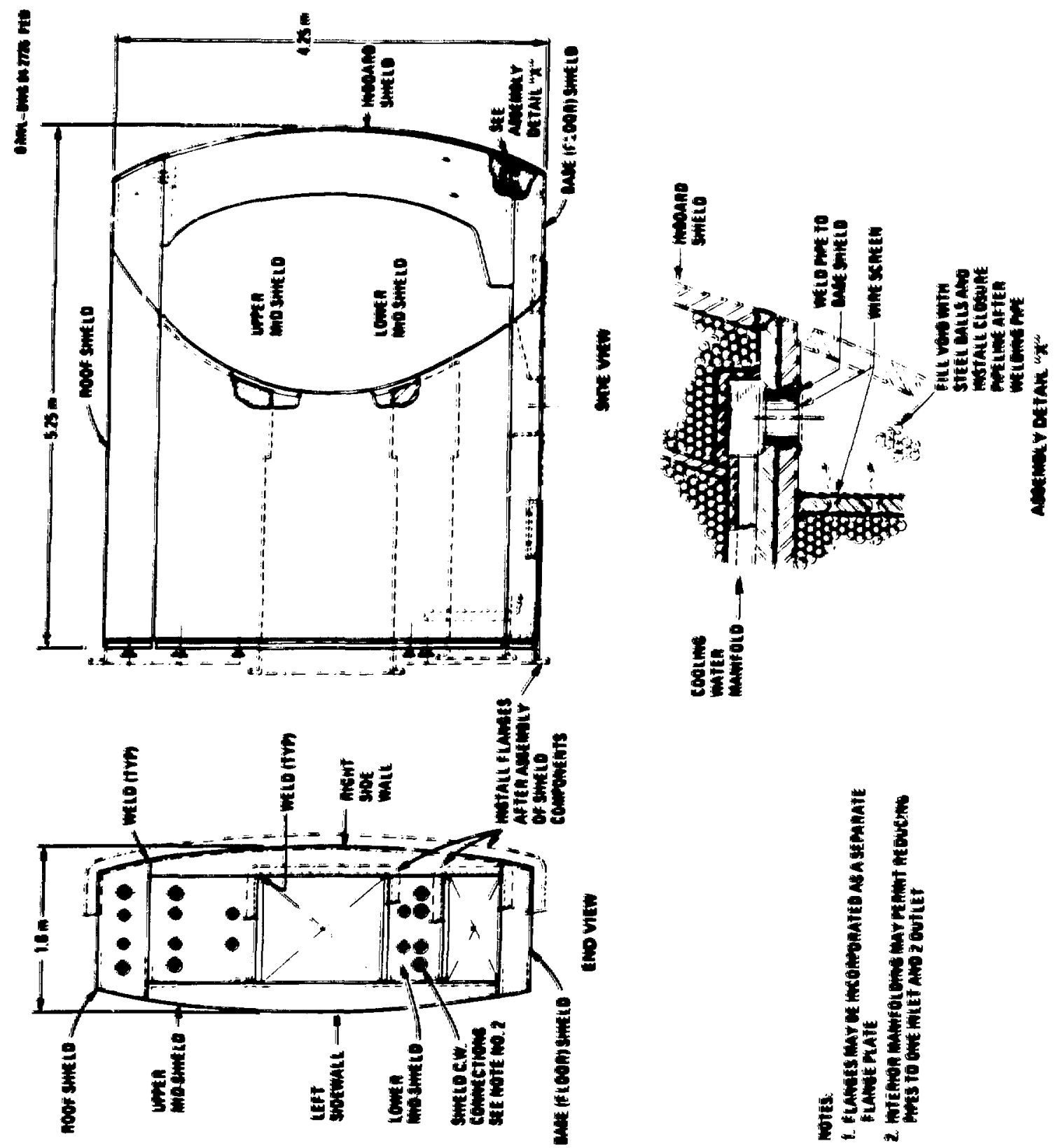


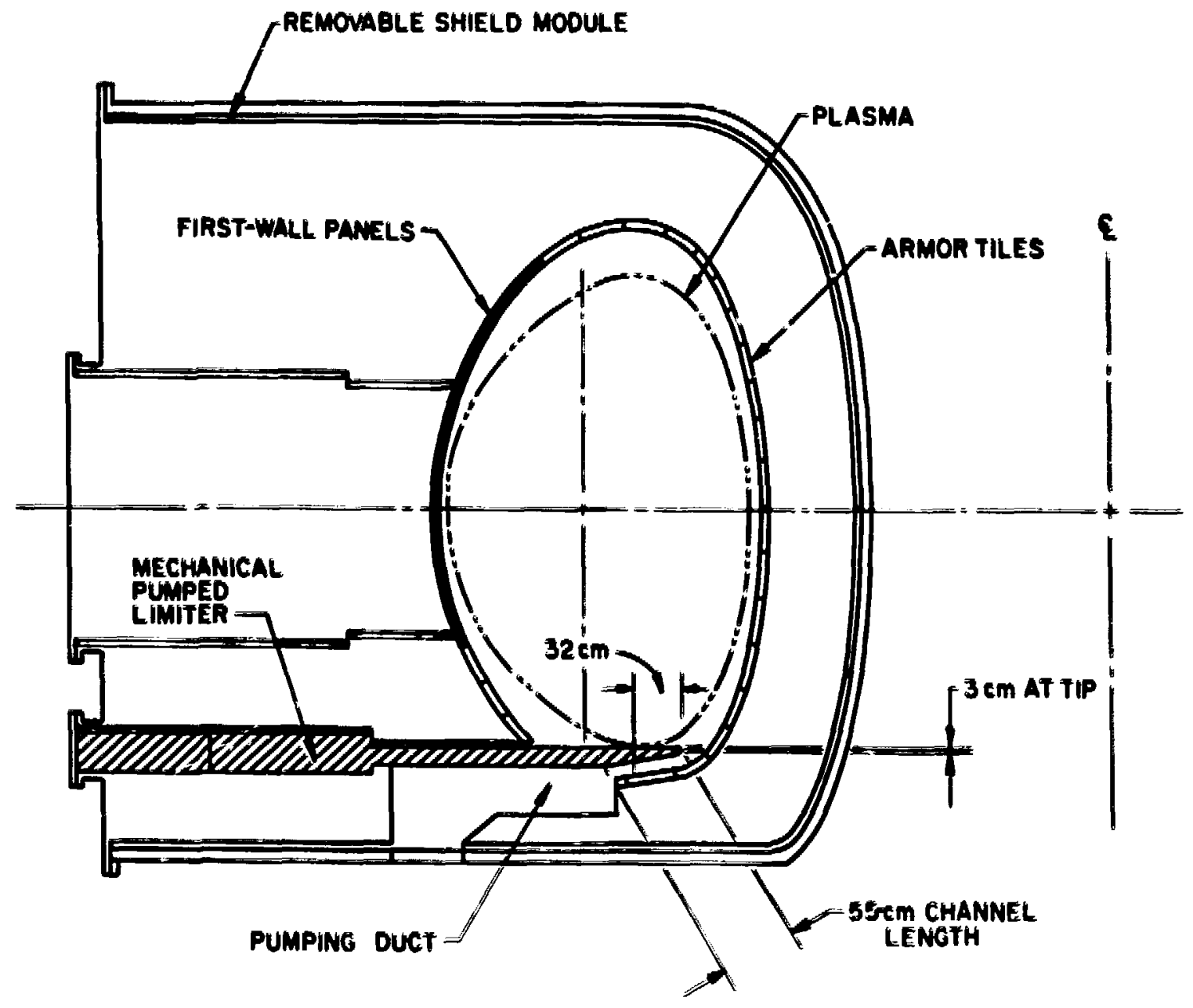



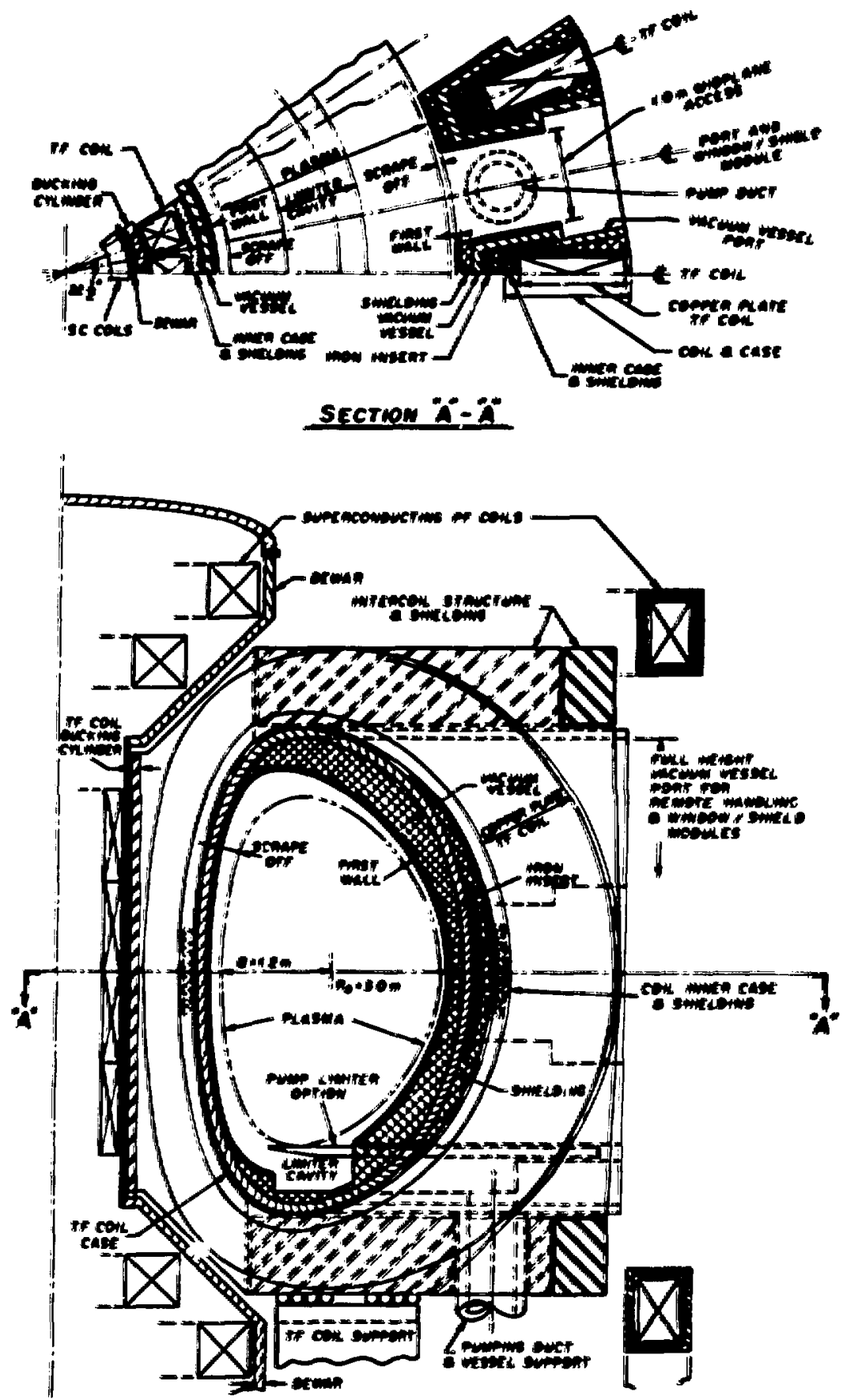

TFC COPPER TF CONFIOURATION 


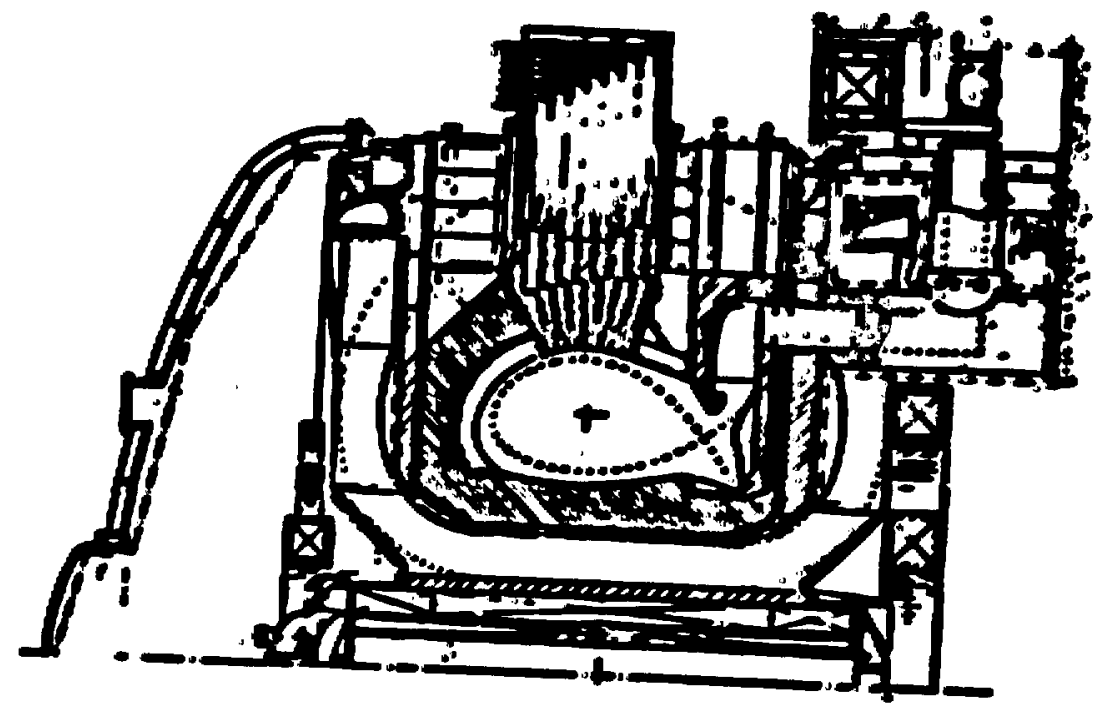

旁

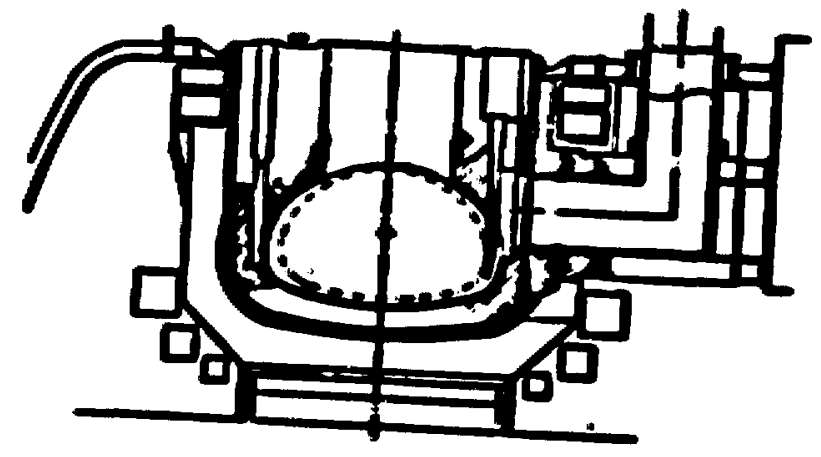

5

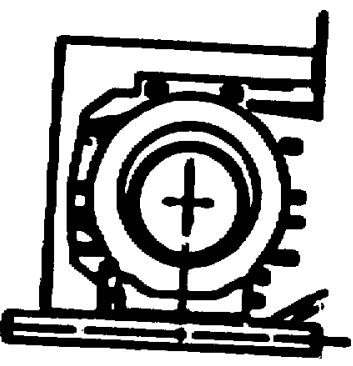

$\frac{1}{16}$ 


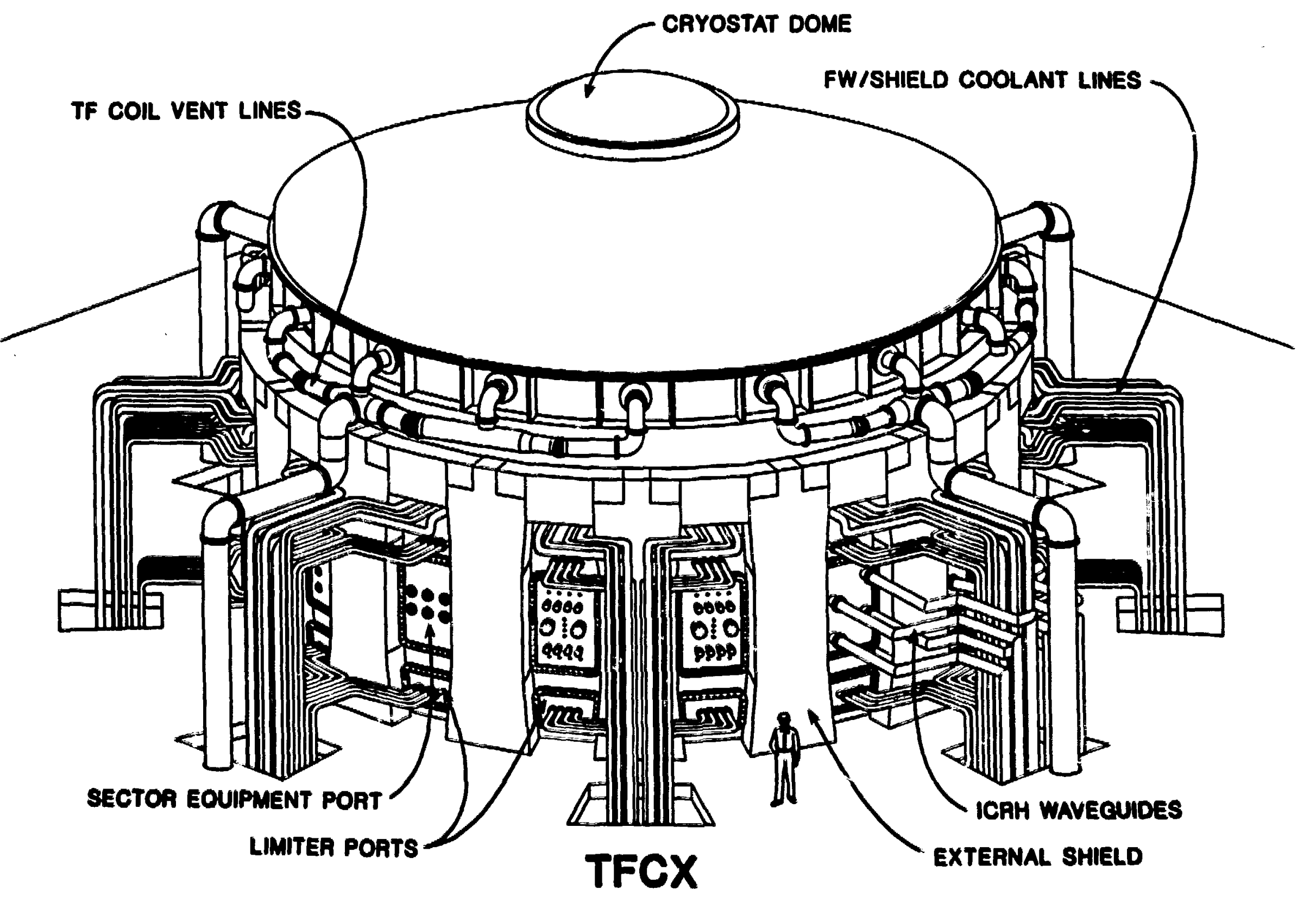




\section{MODULARIZED DESIGN APPROACH}

Vacuum Ring Module

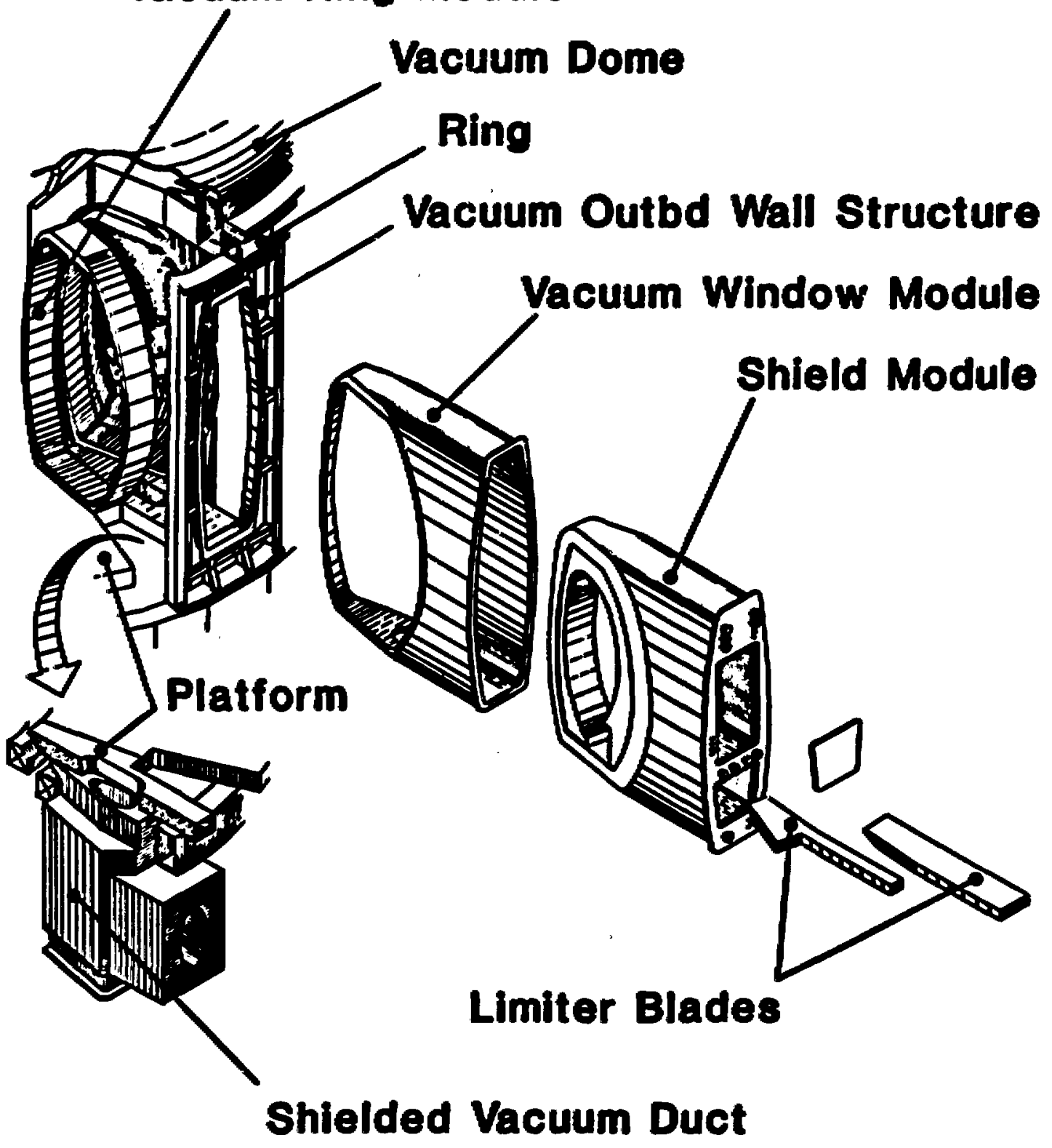




\section{COMDUCTOR COMFIGURATIOH $\mathrm{Nb}_{3} \mathrm{~S}_{\mathrm{N}}$ FORCED FLOW}

$A \times x \times-3400-020-8 k-F E D C$

FEATURES

SAME AS W-LCP BUT WITH THE FOLLOWING MODIFICATIONS

- INCOLOY CONDUIT - REDUCES COOLDOWN STRAIN AND INCREASES CURRENT CARRYING CAPABILITY

- LARGE HELIUM CROSS-SECTIONAL AREA - INCREASES HEAT REMOVAL CAPABILITY AND REDUCES PRESSURE DROP

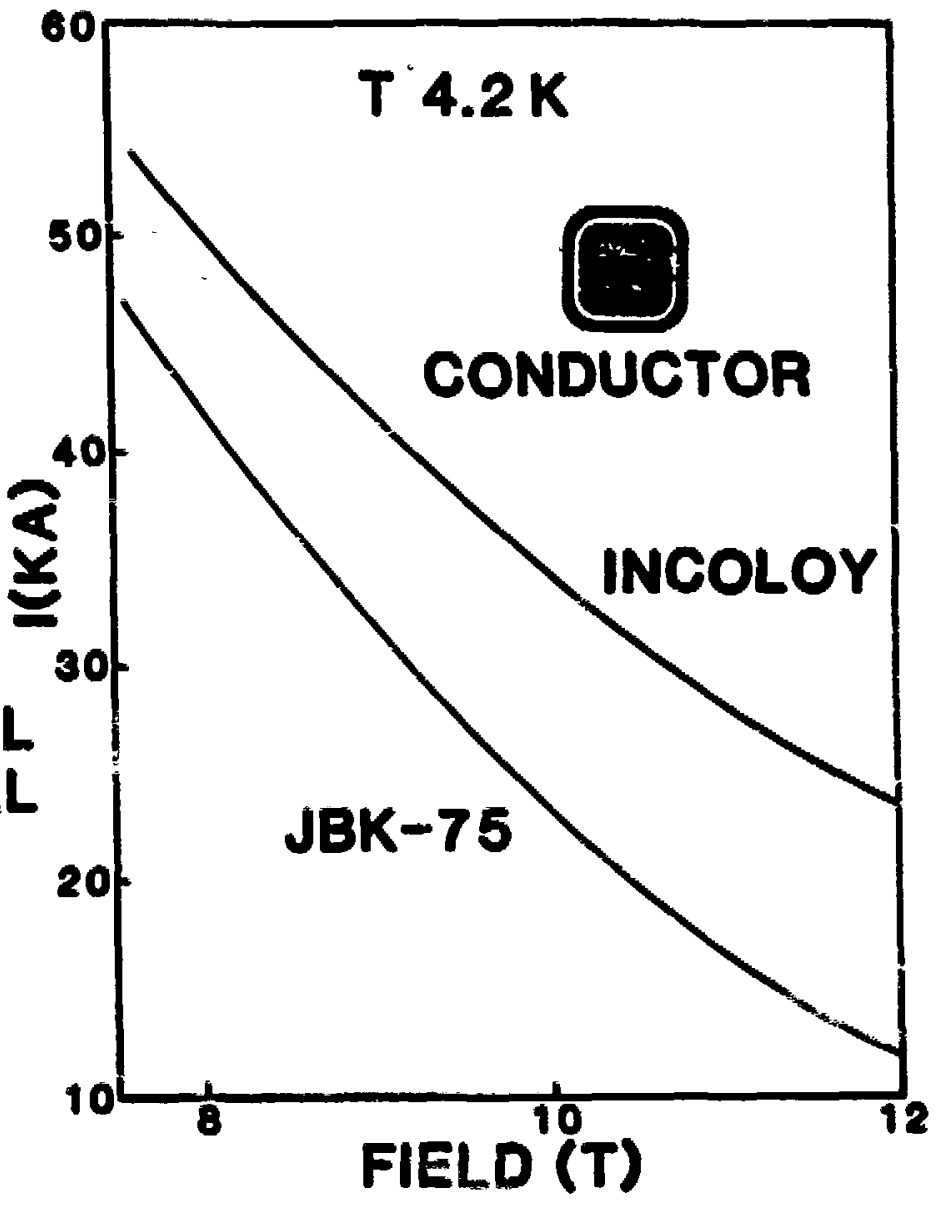




\section{TFCX-S TF WIMDING COMFIGURATIOM}

$A \times x x-8400-000-8 x-F E D C$

Winding Concepts are Feasible

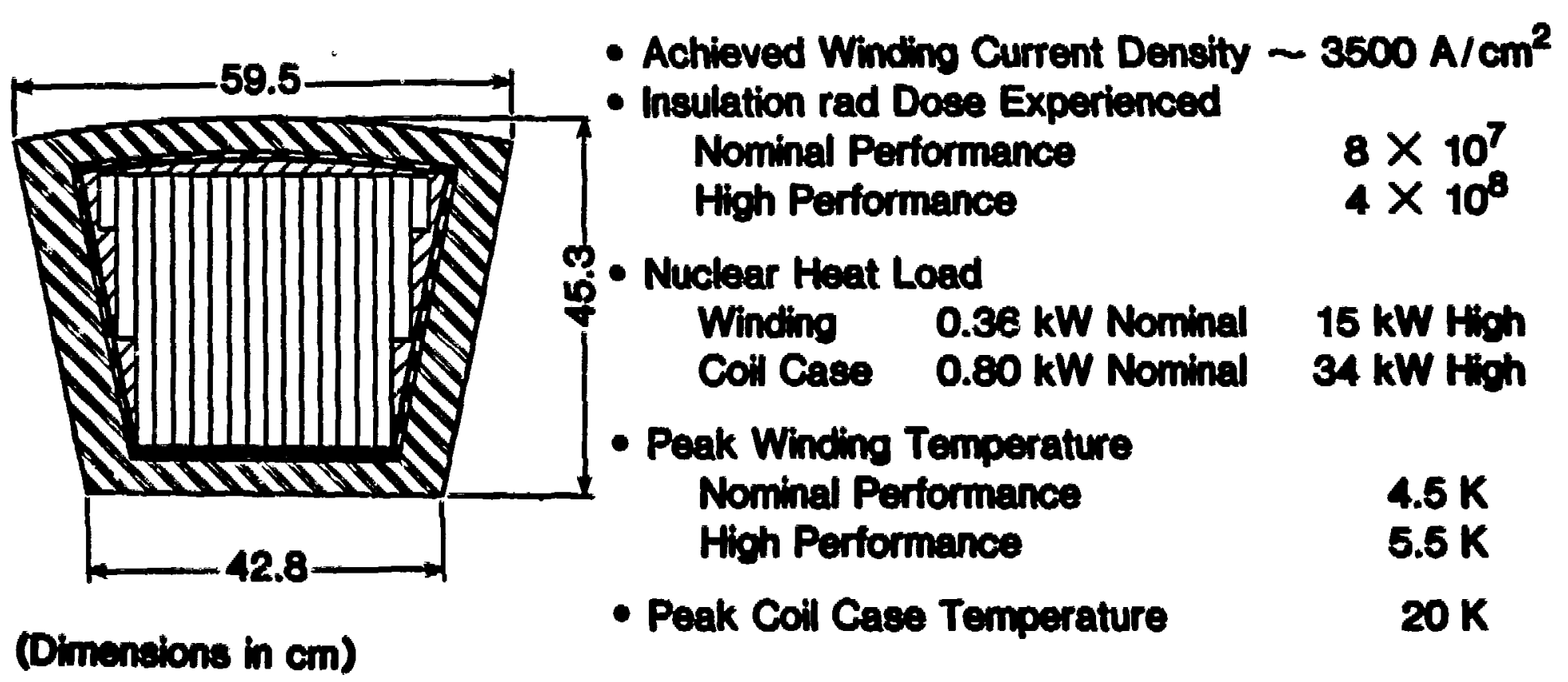




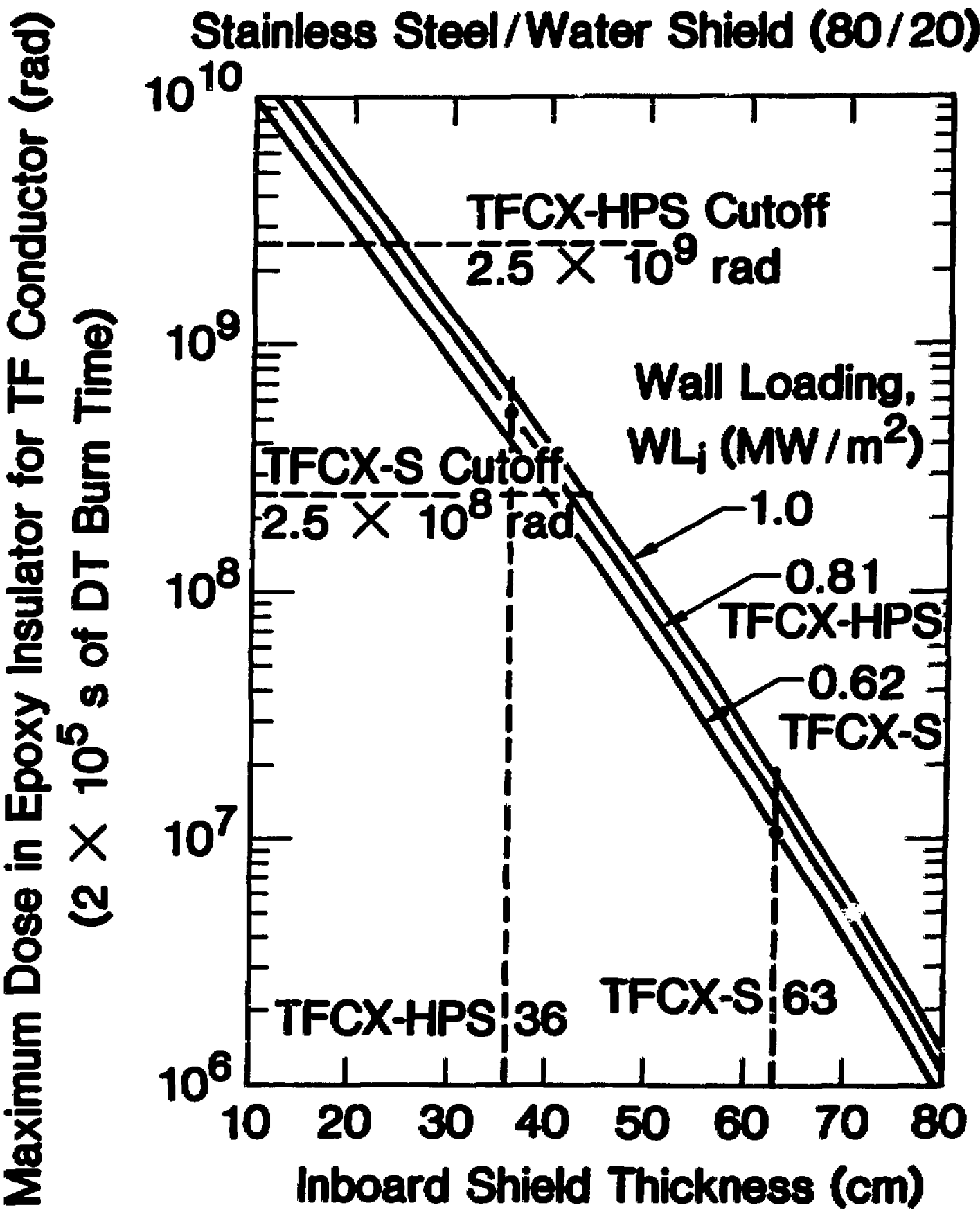




\section{IMBOARD SHIELD}

\section{REQUIREMENTS/PERFORMANCE}

Stainless Steel/Water Shield (80/20)

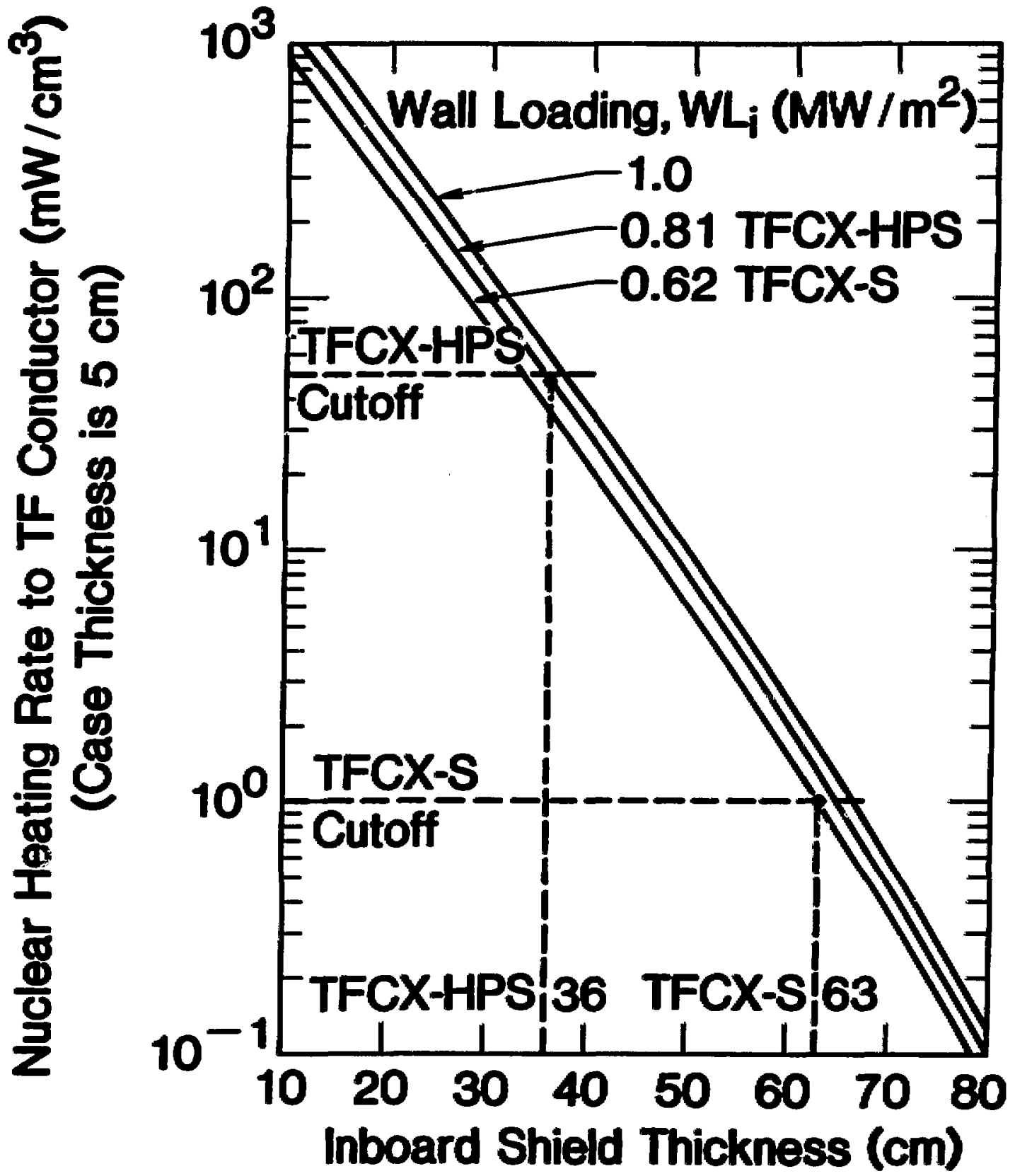




\section{SHIELD POST COMFIGURATION}

$A \times \times \times-8400-0,20-D L-F C D O$

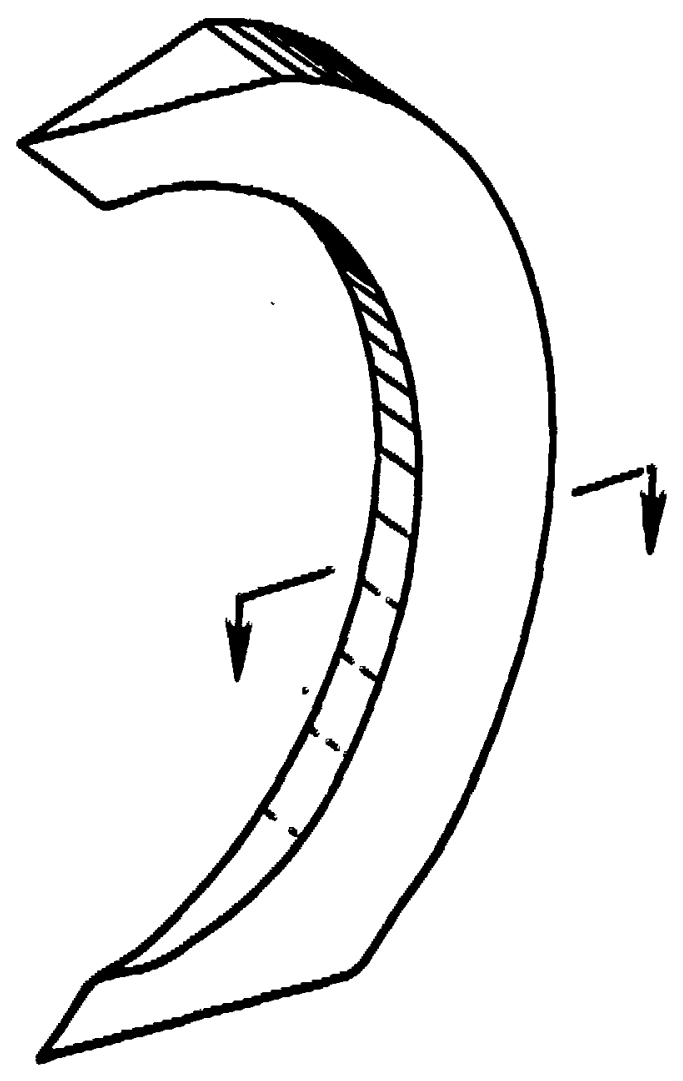

$3 \mathrm{~cm} \mathrm{~B}_{4} \mathrm{C} \rightarrow$

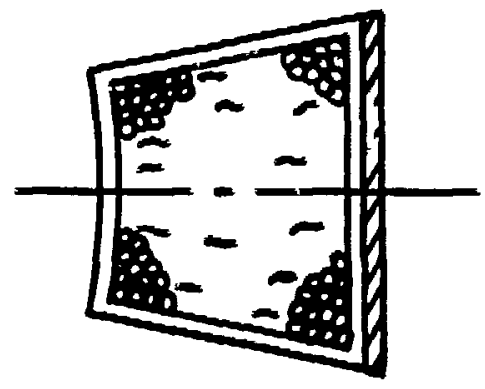

$\frac{\text { MIDPLANE SECTION VIEW }}{60 \% \text { SST Balls/40\% Water }}$ 


\section{REQUIREMENTS}

$A x x x-0400-070-Q F-F E D C$

Performance

Coil Winding, $\mathrm{mW} / \mathrm{cm}^{3}$

Dose to TF Coil Conductor

Insulation (Bonded), rad

Dose to Thermal Insulation

Displacement Damage to Copper

Stabilizer, dpa

Shutdown Dose Rate in Proximity

of Outer Periphery of Device,

mrem/h, $24 \mathrm{~h}$ after Shutdown
Nominal

1

High

Performance

50

$2.5 \times 10^{8} \quad 2.5 \times 10^{9}$

$5 \times 10^{9}$

$2 \times 10^{-4}$

$5 \times 10^{10}$

$2 \times 10^{-4}$ 


\section{CONFIGURATION USED FOR THERMAL/MECHANICAL ANALYSIS}

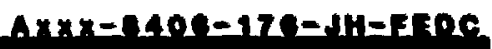

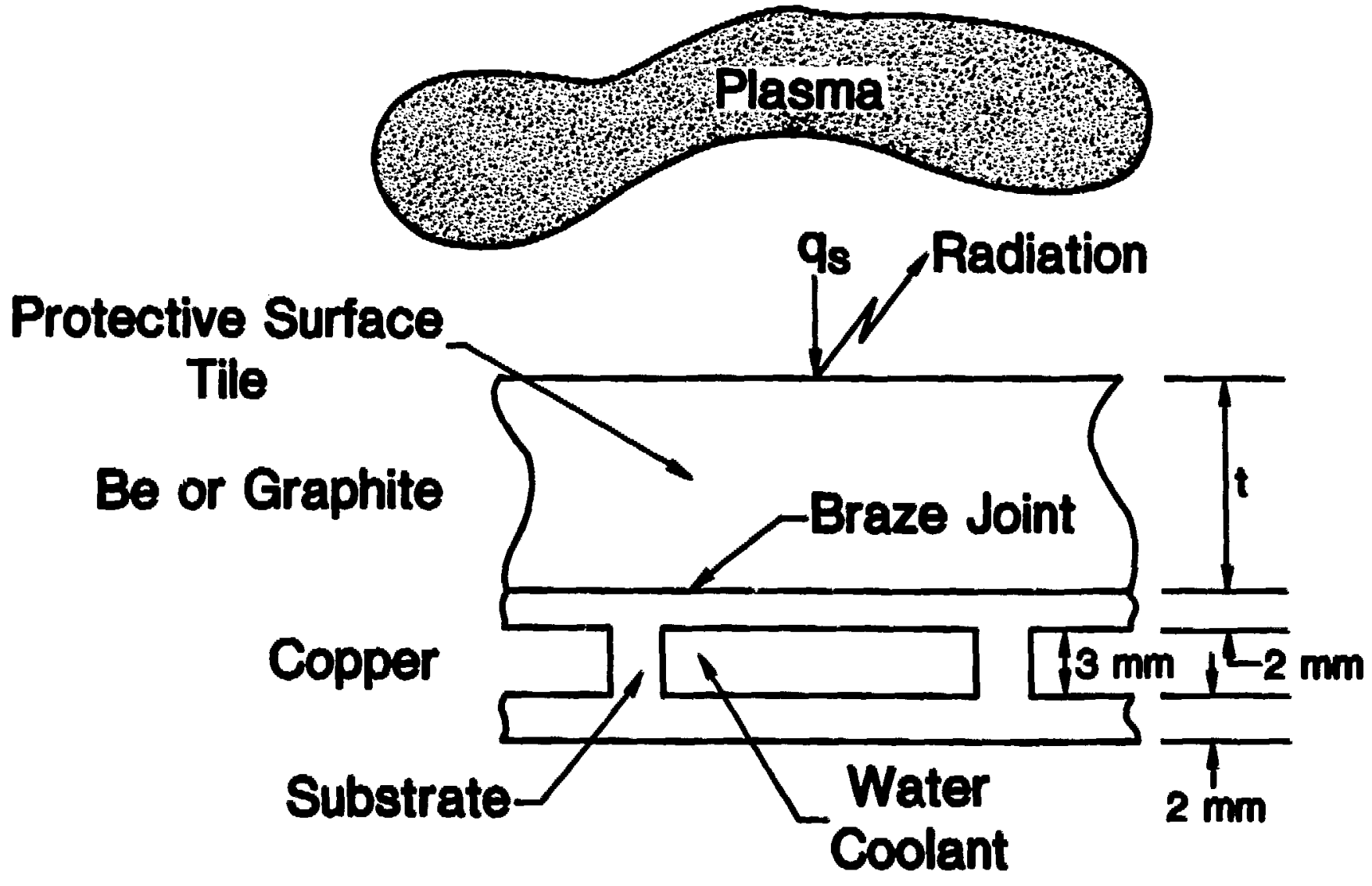




\section{LIMITER DESIGN FEATURES EXPENDABLE PROTECTIIE SURFACE AND REUSABLE CORE}

$A \times x x-0400-100-J H-F E D C$

\section{Protective Surface}

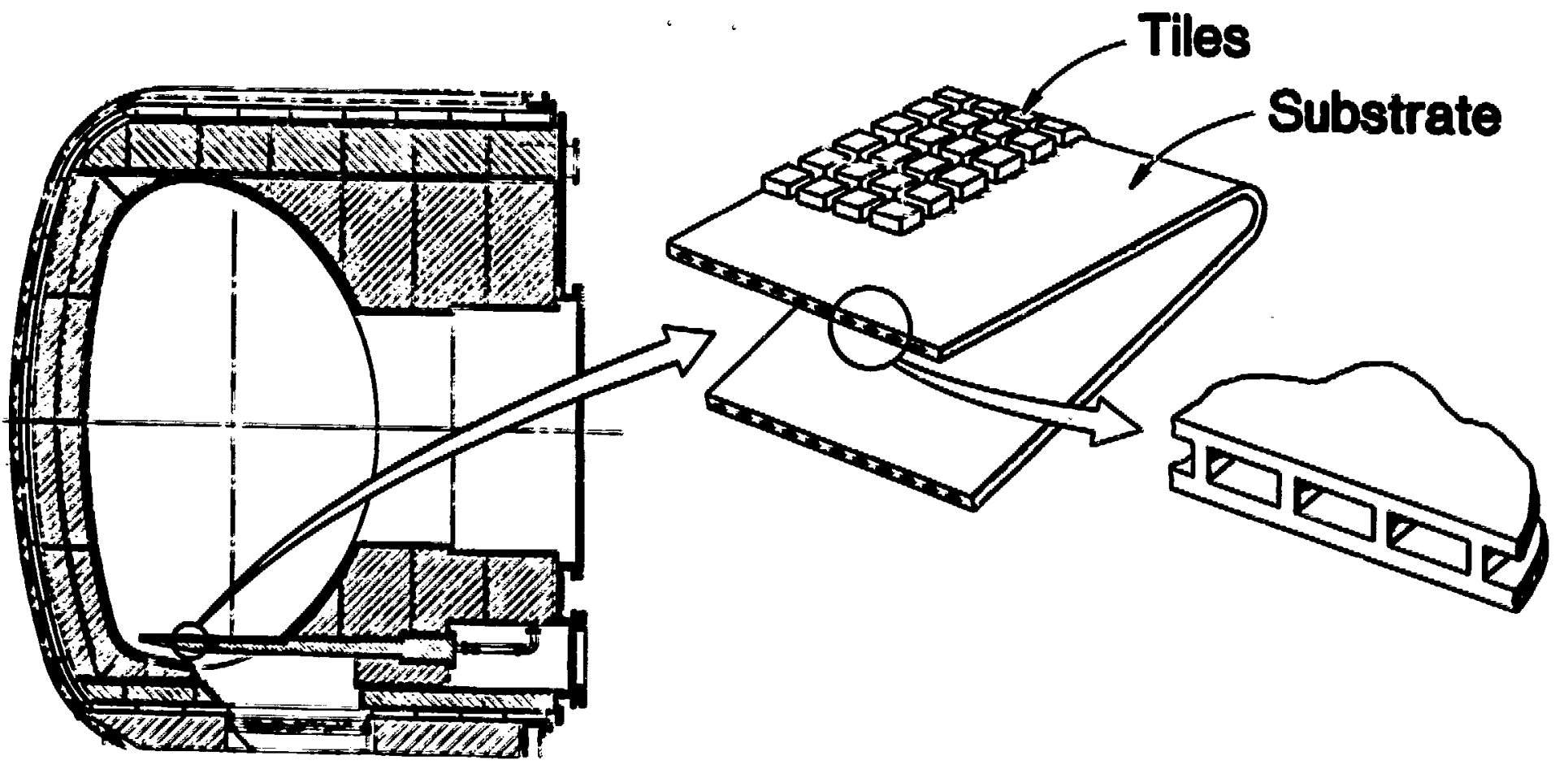




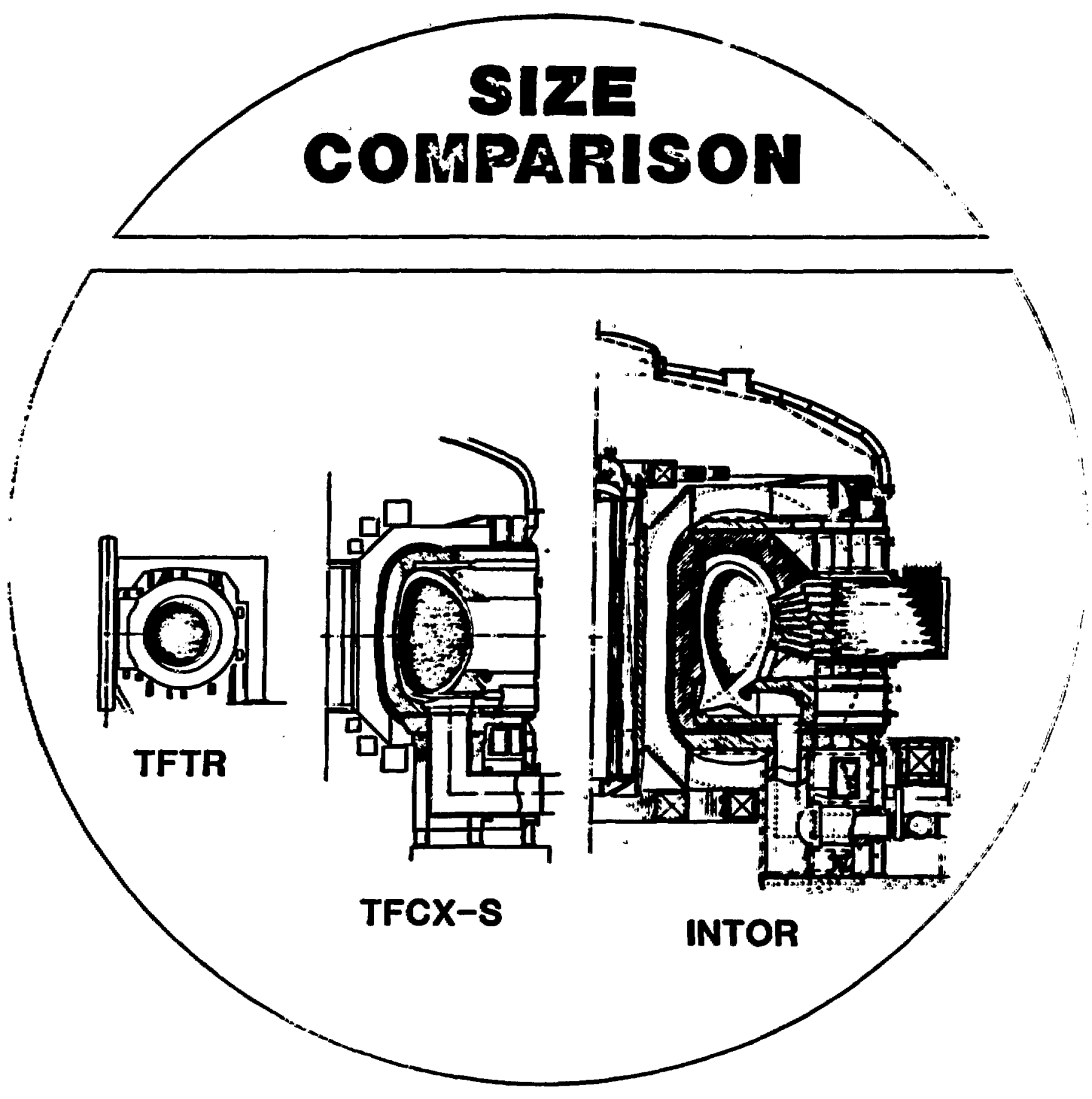



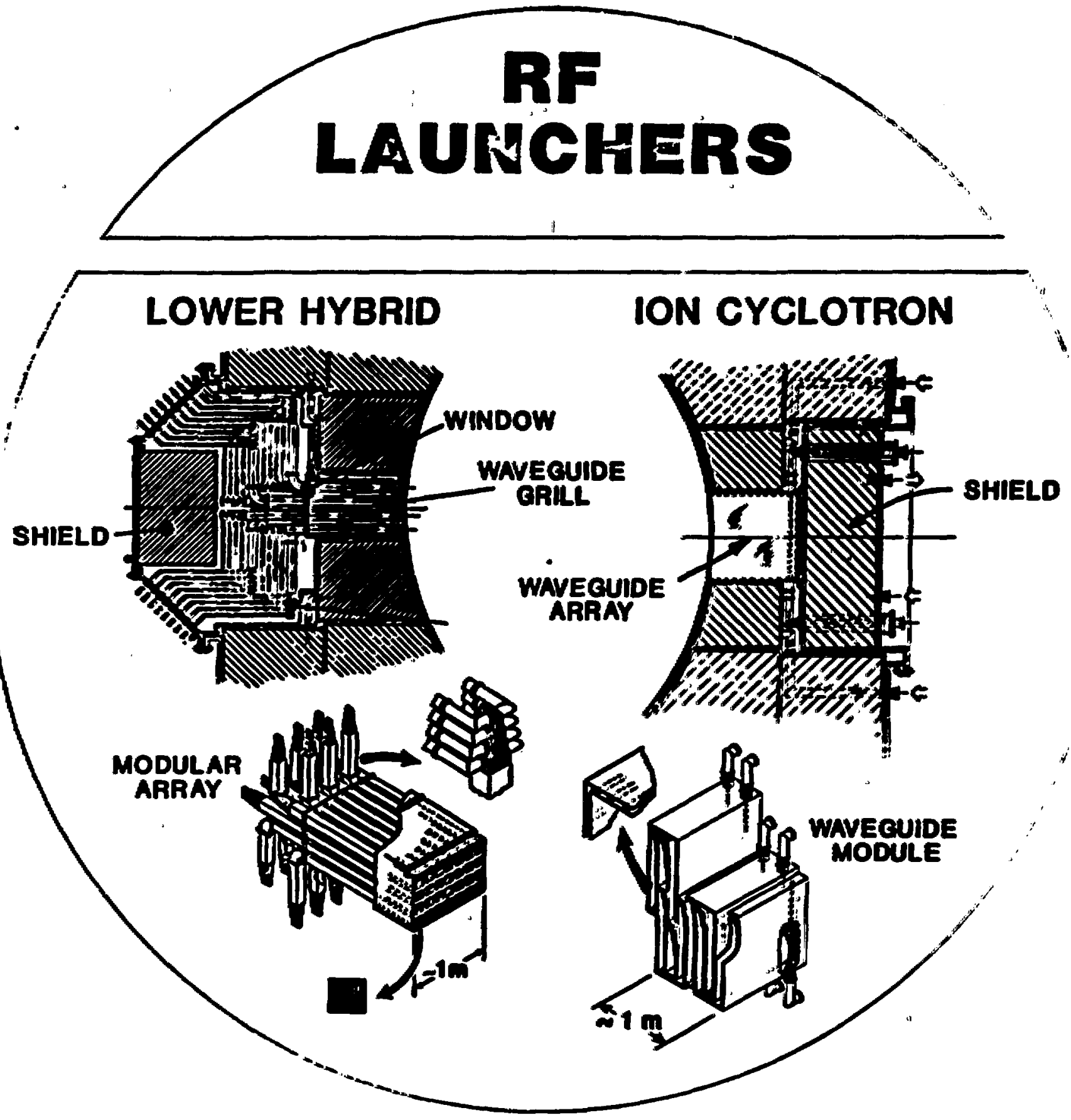


\section{BULK SHIELDING}

Inboard

Intermediate Outboard
DDS 80\% 8sT Plate/20\% Water WIIID 60\% 88T Balle/40\% Water N100 100\% Whater

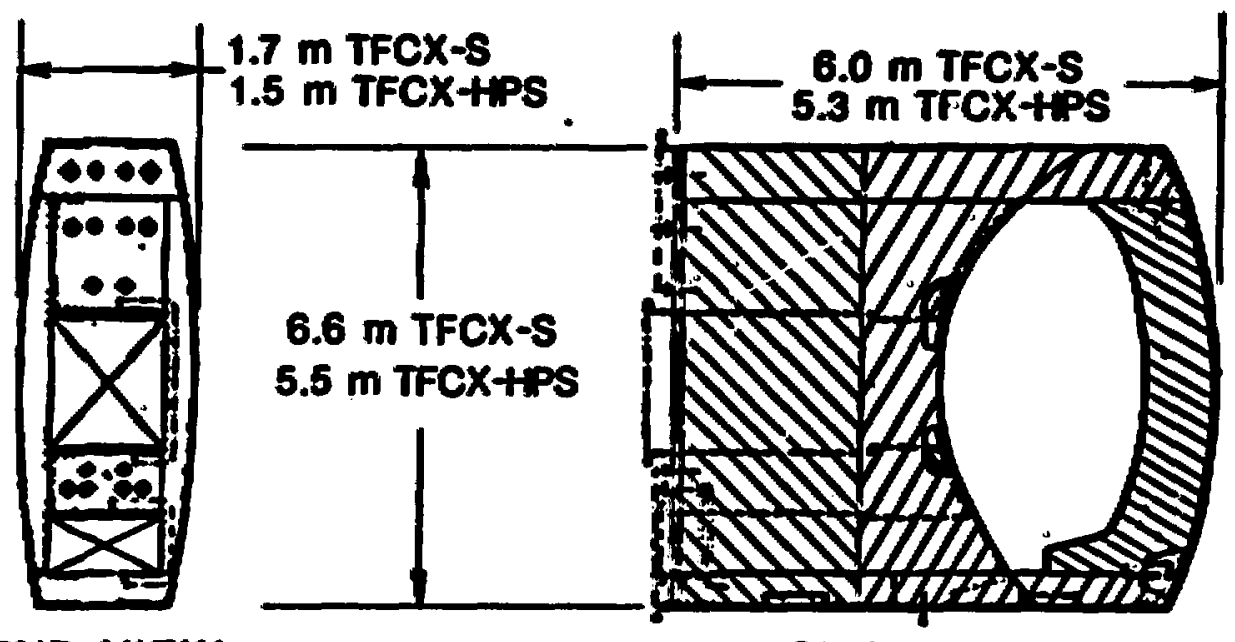

END VIEW

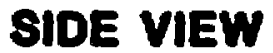




\begin{tabular}{|c|c|c|c|c|}
\hline \multirow[b]{2}{*}{ Matorial } & \multirow[b]{2}{*}{ Temperature (K) } & \multirow[b]{2}{*}{ Application } & \multicolumn{2}{|c|}{ Does Lini (rod) } \\
\hline & & & Nominal & Fith Pertormance \\
\hline Clase-Epoxy (G-10CR) & $\begin{array}{r}300 \\
4\end{array}$ & $\begin{array}{l}\text { Bonded } \\
\text { Unbonded } \\
\text { Bonded } \\
\text { Unbonded }\end{array}$ & $\begin{array}{l}5 E 8 \\
1 E 10 \\
2.5 E 8 \\
\text { SES }\end{array}$ & $\begin{array}{l}E=0 \\
1 E 11 \\
2.6 \pm 0 \\
E=10\end{array}$ \\
\hline ClaserPohyinide & $\begin{array}{r}300 \\
4\end{array}$ & $\begin{array}{l}\text { Bonded } \\
\text { Unbonded } \\
\text { Bonded } \\
\text { Urbonded }\end{array}$ & $\begin{array}{c}\text { PEO } \\
4 E 11 \\
2 E 9 \\
2 E 11\end{array}$ & $\begin{array}{l}4 E 10 \\
1 E 12 \\
2 E 10 \\
E=11\end{array}$ \\
\hline Ceramic $\mathrm{MgN}_{2} \mathrm{O}_{4}$ & 300 & Conting & $>2 \times$ & Pr neutrons/ain \\
\hline
\end{tabular}




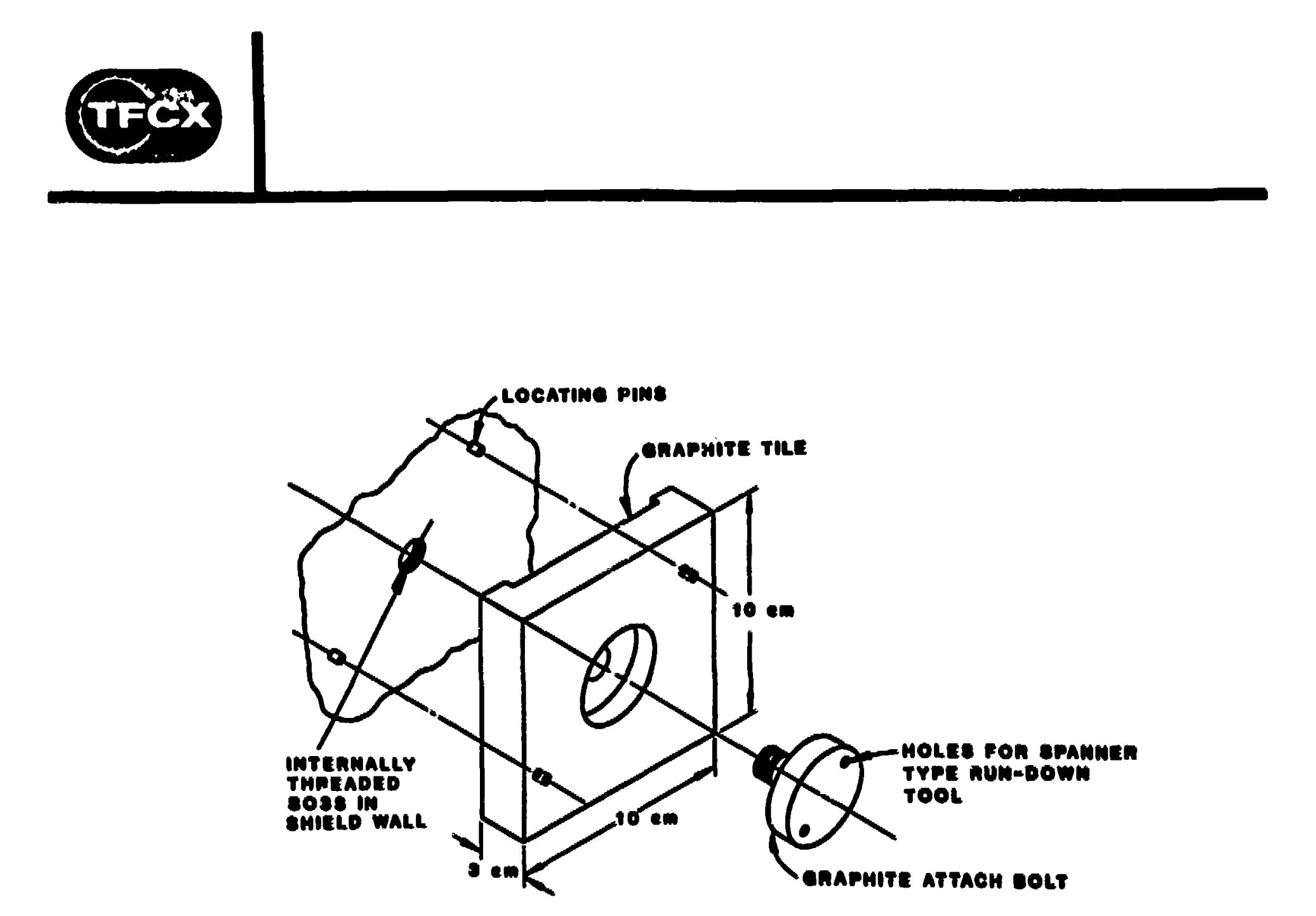




\begin{tabular}{|c|c|c|c|c|}
\hline \multirow[b]{2}{*}{ Paramoter } & \multicolumn{2}{|c|}{ Superconducting } & \multicolumn{2}{|c|}{ Gopper } \\
\hline & Nomina & Hi Port. & Mominal & III Pert. \\
\hline MAJOR RADIUS (m) & 4.08 & 3.61 & 3.36 & 260 \\
\hline MINOR RADIUS $(\mathrm{m})$ & 1.52 & 1.30 & 1.30 & 1.04 \\
\hline ASPECT RATIO & 2.60 & 2.77 & 2.58 & 2.40 \\
\hline FIELD ON AXIS (T) & 3.73 & 4.23 & 4.00 & 50 \\
\hline MBOARD SHIELDNO (m) & 0.62 & 0.36 & 0.12 & 0.018 \\
\hline FUSION POWER (MW) & 267 & 270 & 229 & 197 \\
\hline WALL LOAD $\left(\mathrm{MW} \mathrm{m}^{-2}\right)$ & 0.69 & 0.92 & 0.85 & 1.17 \\
\hline PLASMA CURRENT (MA) & 11.2 & 10.5 & 10.9 & 10.4 \\
\hline PULSE LENGTH (SOC.) & 618 & 452 & 488 & 200 \\
\hline$\frac{\text { LHRF POWER (MW) }}{\text { KRFF POWER InitieVFInal (MW }}$ & in) $\begin{array}{c}32 \\
6 / 31\end{array}$ & $\begin{array}{c}26 \\
10 / 38\end{array}$ & $\begin{array}{c}28 \\
7 / 28\end{array}$ & $\begin{array}{l}18 \\
7 / 26\end{array}$ \\
\hline TF/PF PWR (MW) & - & - & $408 / 81$ & $338 / 100$ \\
\hline BETA (\%) & $\mathbf{5 . 5 1}$ & 5.35 & 8.78 & 8.06 \\
\hline ICN MIRNOV & 1.5 & 1.5 & 15 & 1.6 \\
\hline
\end{tabular}




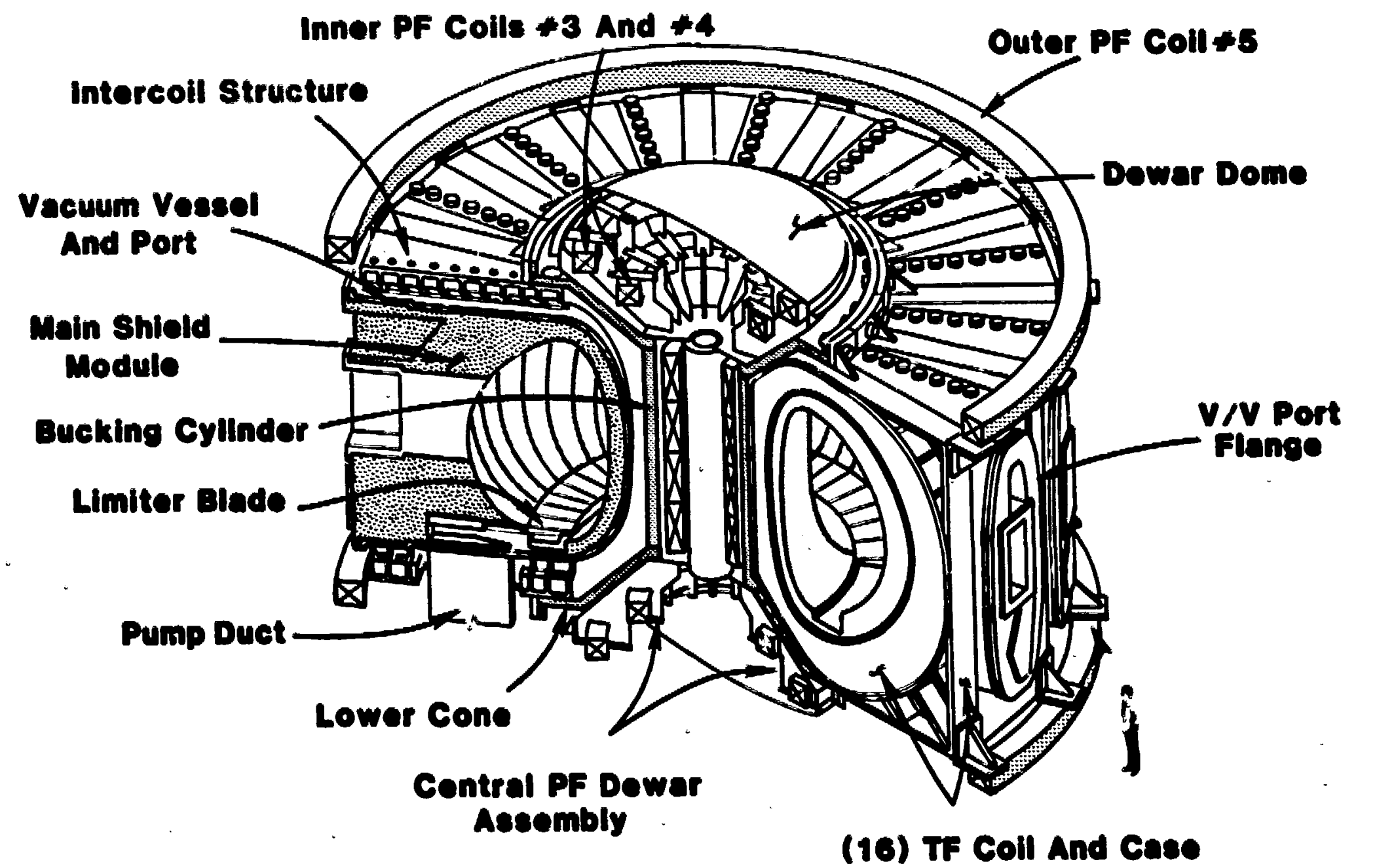




\section{다)}

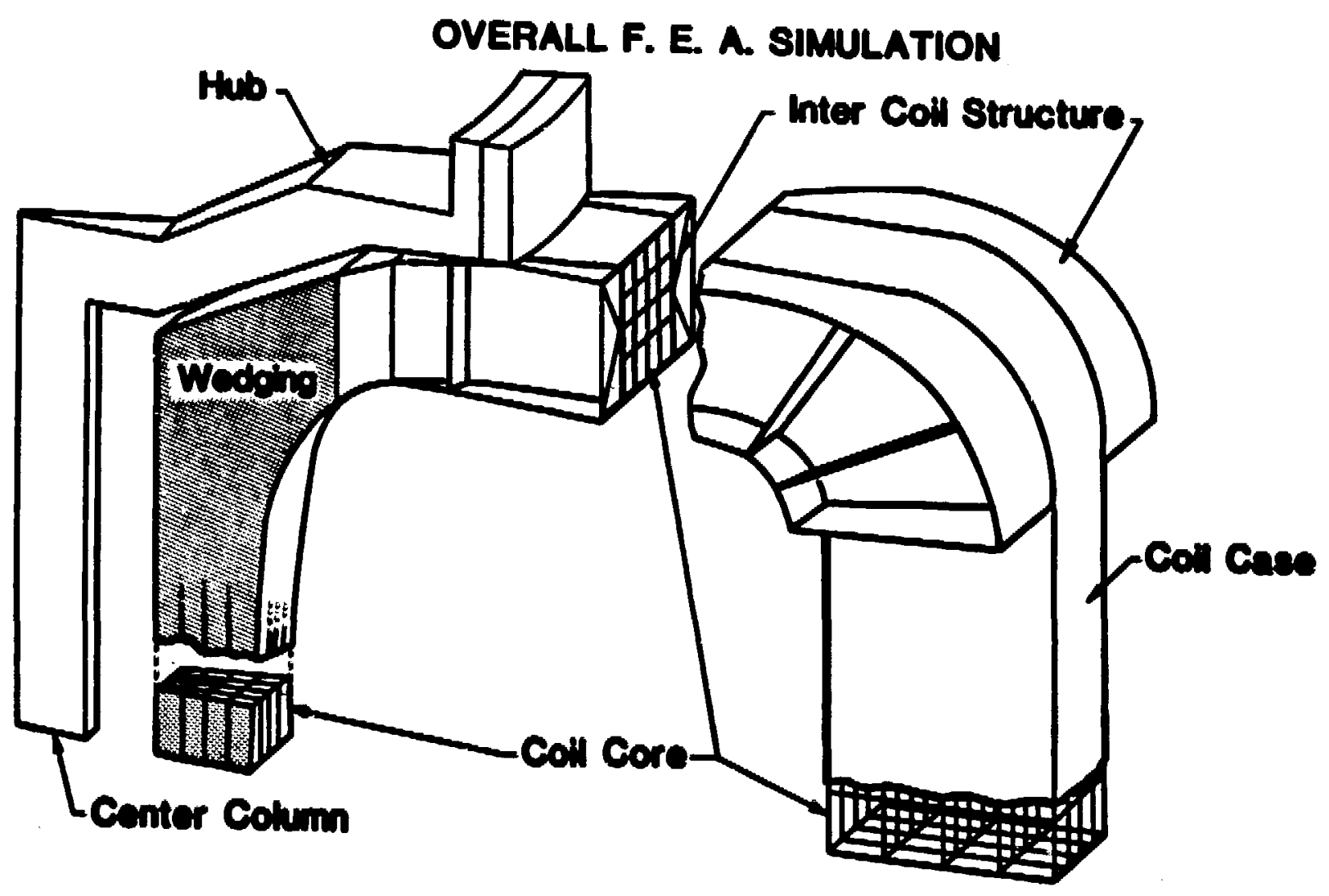

